\title{
Mesalazine (5-aminosalicylic acid) induced chronic hepatitis
}

\author{
P Deltenre, A Berson, P Marcellin, C Degott, M Biour, D Pessayre
}

\begin{abstract}
Background-Treatment of ulcerative colitis or Crohn's disease with sulphasalazine causes several adverse effects, including hepatitis. Sulphasalazine is cleaved by colonic bacteria into 5 -aminosalicylic acid and sulphapyridine. Received wisdom was that 5-aminosalicylic acid was topically active, whereas sulphapyridine was absorbed and caused immunoallergic side effects. Mesalazine, a slow release formulation of 5-aminosalicylic acid, was expected to be a safe alternative. However, several cases of acute hepatitis have been reported.

Case report-A 65 year old man had increased liver enzymes, anti-nuclear and anti-smooth muscle autoantibodies and IgG levels, and lesions of chronic hepatitis after 21 months of mesalazine treatment. Although liver dysfunction had been identified eight months earlier, simvastatin rather than mesalazine had been withdrawn, without any improvement. In contrast, liver enzyme and IgG levels became normal and autoantibodies disappeared after discontinuation of mesalazine administration.

Conclusion-Contrary to initial expectations, mesalazine can cause most of the sulphasalazine induced adverse effects, and hepatic side effects may be almost as frequent. When liver dysfunction occurs, mesalazine administration should be discontinued to avoid the development of chronic hepatitis and liver fibrosis.

(Gut 1999;44:886-888)
\end{abstract}

and Service

d'Hépatologie, Hôpital

Beaujon, 92118 Clichy,

France

P Deltenre

A Berson

P Marcellin

D Pessayre

Service

d'Anatomopathologie, Hôpital Beaujon

C Degott

Centre Régional de Pharmacovigilance, Hôpital Saint-Antoine, 75571 Paris, France M Biour

Correspondence to: Dr D Pessayre, INSERM U481, 92118 Clichy, France.

Accepted for publication 17 December 1998
Keywords: mesalazine; 5 -aminosalicylic acid; adverse drug reaction; hepatotoxicity; chronic hepatitis; liver

Drug combinations are like human couples. When they cause mischief, public opinion is quick to blame the obvious villain. Only after a divorce-or a new drug formulation-has separated these fateful couples do we realise the harmful potential of the "innocent" partner.

A recent example is sulphasalazine (salicylazosulphapyridine). This drug has been used in the treatment of ulcerative colitis and Crohn's disease, despite numerous adverse effects including hepatitis. ${ }^{1}$ Reduction of the sulphasalazine azo bond by colonic bacteria releases sulphapyridine and 5-aminosalicylic acid. ${ }^{1}$ Adverse drug reactions have been attributed to sulphapyridine, and therapeutic activity to 5-aminosalicylic acid. ${ }^{1-4} \mathrm{~A}$ rational answer seemed to be to administer 5-aminosalicylic acid instead. In a formulation (termed mesala- zine) ensuring its slow release in the small and large intestines, 5-aminosalicylic acid was expected to have the therapeutic activity of sulphasalazine (which it does) and to avoid its adverse effects (which unfortunately it does not, or only partly)..$^{5}$

Long held misconceptions die hard, however. Despite reports indicating that 5 -aminosalicylic acid is hepatotoxic, ${ }^{6-8}$ continued belief in its hepatic safety may result in other drugs being suspected when liver dysfunction occurs. We report the case of a patient in whom prolonged administration of mesalazine led to chronic hepatitis and liver fibrosis. This is the first report of mesalazine induced chronic liver disease.

\section{Case report}

A 65 year old man presented on 1 June 1997 with liver dysfunction of unknown cause. $\mathrm{He}$ had been fitted with a pacemaker in 1992 to prevent cardiac arrhythmia, and had been treated with perindopril (4 $\mathrm{mg}$ daily) since 1991, acenocoumarol (4 mg daily) since 1994, and sotalol (160 mg daily) since 1995 (fig 1). In February 1996, he had minor digestive problems and was thought possibly to have Crohn's disease. Mesalazine ( $3 \mathrm{~g}$ daily) combined with Smecta (a diosmectite mucilage, $6 \mathrm{~g}$ daily) administration was started on 15 February 1996. Since March 1996, he had also received four tablets daily of Cirkan (a combination of pancreatic enzymes, ruscus glycosides, ascorbic acid, and hesperidin methyl chalcone used as a venotonic and venoprotective formulation) and $20 \mathrm{mg}$ daily of simvastatin (fig 1).

Serum aspartate aminotransferase (AST) and alanine aminotransferase (ALT) activities were normal in April 1996 (fig 1). On 1 October 1996, serum AST and ALT activities had increased to 9 and 13 times the upper limit of normal $(\mathrm{N})$ respectively (fig 1$)$. There was no alcohol consumption. Hepatitis B surface antigen and anti-hepatitis $\mathrm{C}$ virus antibodies were absent. Physical examination and a pulsed Doppler hepatic sonograph were normal. Simvastatin hepatotoxicity was suspected, and this drug was withdrawn on 15 October 1996, whereas the six other drugs were continued (fig 1).

Eight months later (on 1 June 1997), the patient was referred to our liver unit because of persistent liver dysfunction (fig 1). Serum AST and ALT were at $10 \mathrm{~N}$ and $12 \mathrm{~N}$ respectively, and serum $\gamma$-glutamyl transpeptidase at $2 \mathrm{~N}$. Serum alkaline phosphatase activity and plasma bilirubin were normal. There was no

Abbrevations used in this paper: ALT, alanine aminotransferase; AST aspartate aminotransferase. 


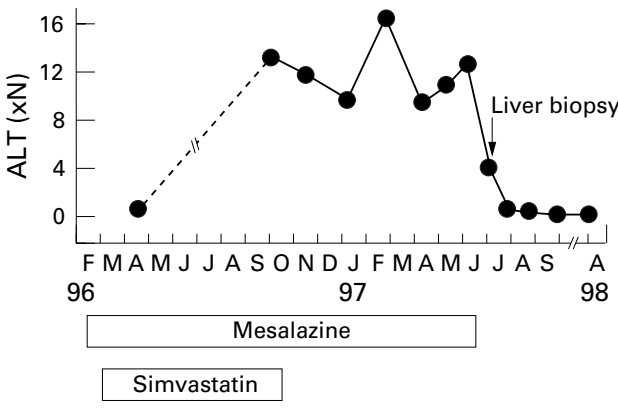

Perindopril, Acenocoumarol, Sotalol, Smecta, Cirkan

Figure 1 Treatments and serum alanine aminotransferase (ALT) activity in a patient with mesalazine induced chronic hepatitis.

rash or fever. Physical examination, hepatic ultrasonography and the haemogram were normal. Serum ferritin was moderately increased $(2 \mathrm{~N})$, but serum iron was normal, as were serum copper, caeruloplasmin and $\alpha 1$ antitrypsin concentrations. Anti-nuclear antibodies (1:500) and anti-smooth muscle antibodies $(1: 200)$ were present, but there were no anti-mitochondrial or anti-liver microsome antibodies. Serum IgG concentration was 25.7 $\mathrm{g} / 1$ (N 7-16 g/l). IgA and IgM levels were normal.

Mesalazine administration was discontinued on 13 June 1997, whereas the five remaining drugs were continued (fig 1). A haemodynamic study and a transjugular liver biopsy were performed three weeks later (on 7 July 1997). The wedge-free hepatic vein gradient $(6 \mathrm{~mm} \mathrm{Hg})$ was moderately increased $(\mathrm{N}, 1-4 \mathrm{~mm} \mathrm{Hg})$.
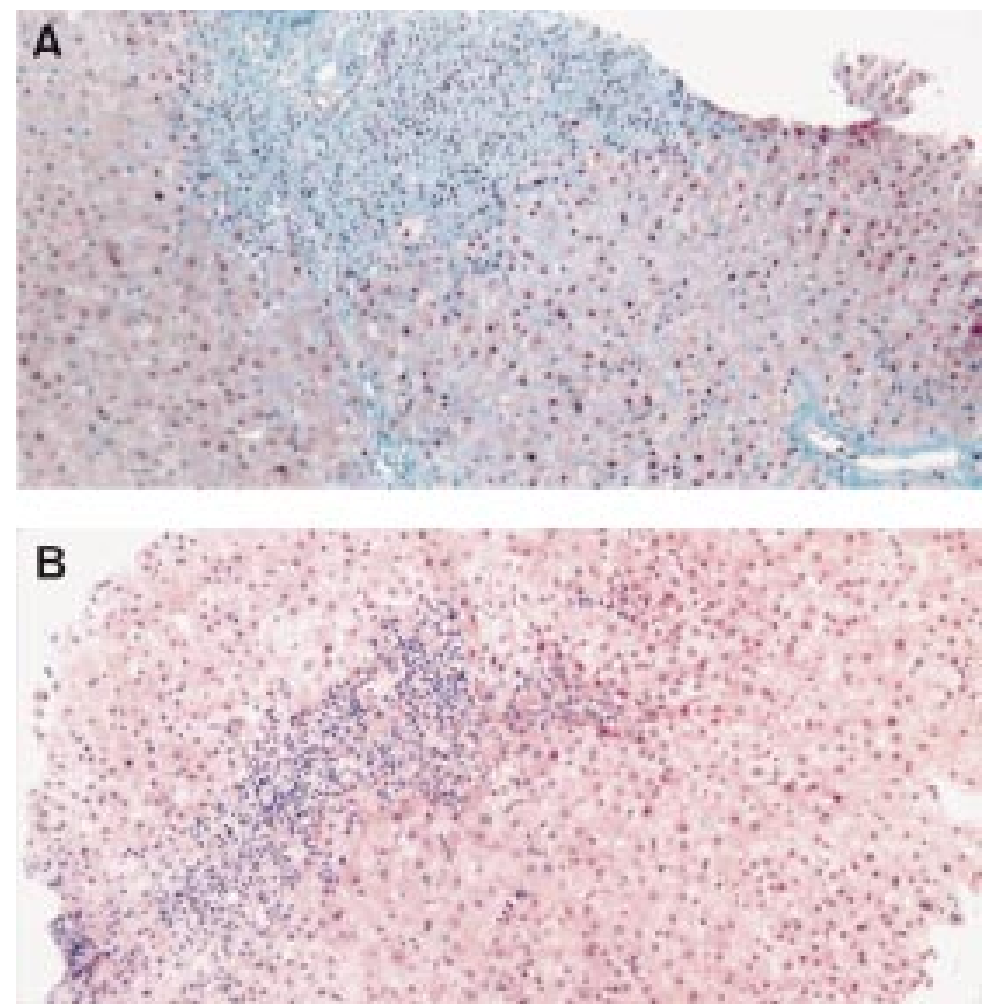

Figure 2 Liver lesions. (A) Mason stain. There is portal and periportal fibrosis, with mild cholangiolar proliferation and mild perisinusoidal fibrosis. (B) Haematoxylin and eosin stain. Fibrous septa contain a mononuclear cell infiltrate and are surrounded by piecemeal necrosis.
There was portal and periportal fibrosis and mild perisinusoidal fibrosis (fig 2A). Portal tract infiltrates consisted mainly of mononuclear cells, with some neutrophils and rare eosinophils. Portal bile ducts were normal, with mild cholangiolar proliferation (fig 2A). Piecemeal necrosis was observed around portal fibrosis (fig 2B). Acidophilic bodies surrounded by inflammatory cells were observed in the hepatic lobule. Mild iron deposits were present in Kupffer cells.

Liver tests quickly improved after interruption of mesalazine administration; they had returned to normal by 12 August 1997 and have remained normal since (fig 1). Antinuclear antibody titres slowly declined (1:500 in June 1997, 1:200 in September 1997, and 1:100 in March 1998), as did anti-smooth muscle antibody titres (1:200 in June and September 1997, 1:50 in March 1998). Antinuclear and anti-smooth muscle antibodies were absent and immunoglobulin levels were normal on 8 September 1998.

Ironically, this patient never had any inflammatory bowel disease.

\section{Discussion}

This patient had raised liver enzyme activity, chronic hepatitis lesions, raised serum IgG levels, and both anti-nuclear and anti-smooth muscle autoantibodies after 21 months of mesalazine treatment. Idiopathic autoimmune hepatitis can be reasonably excluded. Without any immunosuppressive therapy, liver tests and serum IgG became normal; autoantibodies disappeared, and the liver disease never relapsed. Instead, mesalazine probably caused chronic hepatitis and autoimmune manifestations in this patient. No other cause of liver disease was detected. Although liver tests were normal before mesalazine administration, they increased during mesalazine use, remained abnormal as long as mesalazine was continued, and quickly improved once mesalazine was withdrawn (fig 1). Although the patient had received six other drugs, simvastatin was initially withdrawn without any improvement, and the five remaining drugs did not prevent recovery once mesalazine was discontinued (fig 1).

Mesalazine was developed as a safe alternative to sulphasalazine. The latter causes diverse side effects, including fever, rash, pancreatitis, pneumonitis, atypical lymphocytosis, reversible oligospermia, neuropathy, and hepatitis. ${ }^{2-4} \mathrm{Be}-$ cause these adverse effects resemble the immunoallergic side effects of sulphonamides, and only the sulphapyridine (sulphonamide) moiety of sulphasalazine was thought to be significantly absorbed, it was believed that sulphapyridine caused adverse effects, while 5 -aminosalicylic acid was topically active..$^{1-4}$

It therefore came as a surprise when mesalazine (a slow release formulation of 5-aminosalicylic acid) was shown to cause fever, ${ }^{5}$ rash, ${ }^{5}$ eosinophilia, ${ }^{5}$ renal injury, ${ }^{1}$ liver dysfunction, ${ }^{5-8}$ myocarditis, ${ }^{9}$ neuropathy, ${ }^{10}$ and pancreatitis. ${ }^{11}$ In a randomised trial of coated mesalazine (115 patients) versus sulphasalazine (105 patients), the total incidence of adverse drug reactions was less with mesalazine 
(14\%) than with sulphasalazine (24\%), but hepatitis was almost as frequent: mesalazine caused liver injury in three patients, and sulphasalazine in four. ${ }^{5}$ In 11 published clinical trials mentioning liver test abnormalities, the global incidence of mesalazine induced liver dysfunction was $2.8 \% .^{12}$

In addition to the cases of liver dysfunction mentioned in clinical trials, eight individual cases of mesalazine induced hepatitis have been published, ${ }^{12}$ including three biopsy proven cases. $^{6-8}$ The latter included a woman who initially had a skin rash during sulphasalazine administration, and later developed fever, rash, atypical lymphocytosis, and liver cell necrosis after she was switched to mesalazine. ${ }^{6}$ Mesalazine induced hepatitis has occurred six days to one year after the onset of the treatment. ${ }^{12}$ In the seven patients in which this could be assessed, hepatitis exhibited a cytolytic liver test profile in four patients, a mixed pattern in one, and a cholestatic pattern in two. ${ }^{12}$ In two patients, the liver injury was associated with immunoallergic manifestations. ${ }^{6}$ All previously reported patients had acute hepatitis. ${ }^{12}$ In contrast, our patient exhibited chronic liver disease, most probably because mesalazine administration was continued for eight months after initial detection of the liver injury.

Mesalazine administration may cause higher 5-aminosalicylic bioavailability than sulphasalazine administration, ${ }^{13}$ increasing the probability of 5-aminosalicylic acid induced adverse effects. Therefore recognition of the hepatotoxicity of mesalazine does not necessarily indicate that sulphapyridine was not involved in sulphasalazine induced hepatitis. Nevertheless, the similitude of adverse effects is very striking, and it may be speculated that at least some sulphasalazine induced adverse effects were actually caused by 5 -aminosalicylic acid.

The mechanism responsible for 5-aminosalicylic acid induced liver injury has not been determined. A possible explanation is metabolic activation to covalent binding species causing immunisation. This mechanism should be investigated, as it may help to disclose genetic, nutritional, or therapeutic factors predisposing to mesalazine induced liver injury.
Interestingly, our patient did not have Crohn's disease or ulcerative colitis, indicating that inflammatory bowel disease is not required for mesalazine hepatotoxicity.

Because Crohn's disease is a serious condition, one may be tempted to continue mesalazine administration despite incipient liver dysfunction. The present case should serve as a warning that this may cause chronic liver disease.

In conclusion, mesalazine has not fully lived up to initial expectations. Although this drug was developed as a safe alternative to sulphasalazine, it may, in fact, reproduce most of the adverse effects that had been attributed to the sulphapyridine moiety of suphasalazine. When liver dysfunction occurs, mesalazine should be discontinued to avoid the development of chronic hepatitis and liver fibrosis.

This work was supported in part by The European Union BIOMED 2 Eurohepatox Programme (Contract BMH4CT96-0658), the Programme Hospitalier de Recherche Clinique 95-96, and the Réseau Hépatox.

1 Hayllar J, Bjarnason I. Suphasalazine in ulcerative colitis: in memoriam? Gut 1991;32:462-3.

2 Das KM, Eastwood MA, McManus JPA, et al. Adverse reactions during salicylazosulfapyridine therapy and the relation with drug metabolism and acetylator phenotype. $N$ Engl F Med 1973;289:491-5.

3 Sotolongo RP, Neefe LY, Rudzki C, et al. Hypersensitivity reaction to sulfasalazine with severe hepatotoxicity. Gastroenterology 1978;75:95-9.

4 Farrell G. Drug-induced liver disease. London: ChurchillLivingstone, 1994.

5 Rachmilewitz D. Coated mesalazine (5-aminosalicylic acid) versus sulphasalazine in the treatment of active ulcerative colitis: a randomized trial. BMF 1989;298:82-6.

6 Hautekeete ML, Bourgeois N, Potvin P, et al. Hypersensitivity with hepatotoxicity to mesalazine after hypersensitivity to sulfasalazine. Gastroenterology 1992;103:1925-7.

7 Coumaros D, Georges C, Zerbe S. Salicylazosulfapyridine SASP) hepatitis: role of 5-aminosalicylic acid (5-ASA). Scand $\mathcal{F}$ Gastroenterol 1989;24(suppl 158):132-3.

8 Stochus B, Meybehm M, Spengler U, et al. Cholestasis associated with mesalazine therapy in a patient with Crohn's disease. F Hepatol 1997;26:425-8.

9 Kristensen KS, Hoegholm A, Bohr L, et al. Fatal myocarditis associated with mesalazine. Lancet 1990;335:605.

10 Woodward DK. Peripheral neuropathy and mesalazine. BMF 1989;229:1224.

11 Deprez P, Descamps C, Fiasse R. Pancreatitis induced by 5-aminosalicylic acid. Lancet 1989;2:445-6.

12 Biour M, Poupon R, Grange JD, et al. Hépatotoxicité des médicaments. Onzième mise à mise à jour du fichier bibliographique des atteintes hépatiques et des médicaments responsables. Gastroenterol Clin Biol (in press).

13 Rasmussen SN, Bondesen S, Hvidberg EF, et al. 5-Aminosalicylic acid in slow release preparation: bioavailability, plasma level, and excretion in humans. Gastroenterology 1982;83:1062-70. 